\title{
Chaotic Behaviour of Photonic Crystals Resonators
}

\author{
Di Falco A. ${ }^{a}$, Liu C. ${ }^{b}$, Krauss T. F ${ }^{c}$, and Fratalocchi A. ${ }^{b}$ \\ ${ }^{a}$ SUPA, School of Physics and Astronomy, University of St Andrews, North Haugh, St \\ Andrews, KY16 9SS, UK \\ ${ }^{b}$ PRIMALIGHT, Faculty of Electrical Engineering; Applied Mathematics and Computational \\ Science, King Abdullah University of Science and Technology (KAUST), Thuwal 23955-6900, \\ Saudi Arabia \\ ${ }^{c}$ Department of Physics, University of York, Heslington, York, YO10 5DD, UK
}

\begin{abstract}
We show here theoretically and experimentally how chaotic Photonic Crystal resonators can be used for energy harvesting applications and the demonstration of fundamental theories, like the onset of superradiance in quantum systems.
\end{abstract}

Keywords: Chaotic resonators, Photonic Crystals, Optical resonators

\section{INTRODUCTION}

The efficiency of an optical resonator, also known as quality factor (Q), is determined by the ratio of the stored electromagnetic energy and the power dissipated through all the possible loss channels, at a given frequency. The physical mechanism used to trap light is specific to each resonator. Q is related to the characteristic decay time of energy of the resonator, identified by the lifetime $\tau=2 Q / \omega$. The higher $\mathrm{Q}$, the longer the lifetime of the modes at a given frequency $\omega$. In the frequency domain, higher Qs are associated to narrow resonances. Although most applications benefit of the highest possibly Qs, e.g. to fabricate sensors, delay lines, lasers, tunable filters and in general to promote a strong interaction between light and matter ${ }^{1-4}$ there exists a compromise between bandwidth of the resonator and energy storage. Q and the lifetime are directly linked to the ability to couple light to the resonator from external channel, which is related to the suitability of the resonator as light harvesting device. The maximum amount of power transfer from an external source onto the resonator is obtained in condition of impedance matching, when the internal lifetime of the resonator (as measured for an isolated resonator) is identical to external one (rate of energy exchange with in- and out-coupler channels). Most multimodal resonators achieve this condition only for a specific set of frequencies. This means that for a broadband source most of the frequencies are not efficiently coupled to the resonator. Several schemes have been suggested in literature to overcome this limitation, e.g. based on tailored impedance matching for multiple wavelength ${ }^{5}$ or using the splitting of the modes due to the coupling of identical resonators, to broaden the overall response of the system. ${ }^{6}$ Alternative approaches engineer the efficiency of diffracting gratings to enhance the absorption in a thin film, ${ }^{7}$ or use iterative numerical optimisation to identify complex topologies of resonators that achieve high harvesting efficiency. ${ }^{8}$

Here we present a class of photonic cavities that exploit chaos to trap efficiently light, by fulfilling the impedance matching requirements for a broad range of wavelengths. To this extent we adopt Photonic Crystals (PhCs) resonators based on silicon-on-insulator platform in the near infrared range. The choice is motivated by the fact that $\mathrm{PhCs}$ resonators can be defined with an arbitrary topology and that they enable an extremely accurate control of the loss channels. In the following sections we first describe the fabrication and characterisation of chaotic $\mathrm{PhC}$ resonators, we then focus on their broadband energy harvesting properties and we conclude with a suggestive example of how this class of resonators can be used to address fundamental properties of light matter interaction, like the onset of superradiance.

Further author information: (Send correspondence to A. Di Falco)

E-mail: adf10@st-andrews.ac.uk

Quantum Sensing and Nanophotonic Devices XII, edited by Manijeh Razeghi, Eric Tournié, Gail J. Brown, Proc. of SPIE Vol. 9370, 93700R · (C) 2015 SPIE · CCC code: 0277-786X/15/\$18 · doi: 10.1117/12.2077730 

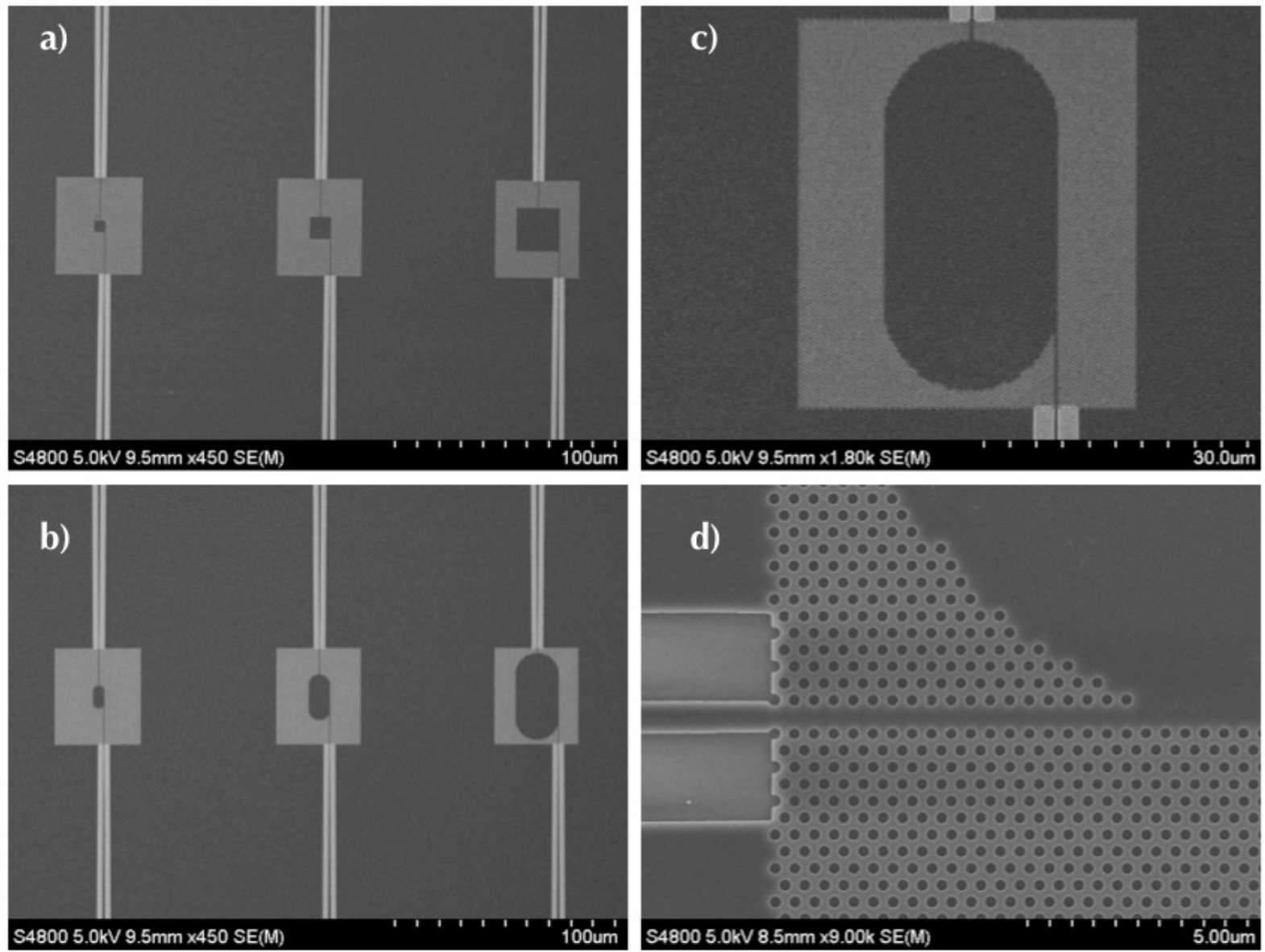

Figure 1. a) and b) SEM figures of square and stadium cavities respectively. c) and d) enlarged views of a stadium resonator and coupling region.

\section{SAMPLE FABRICATION AND OPTICAL CHARACTERIZATION}

All the samples referred to in this paper are realised in Silicon on Insulator (SOI) platform (220nm thick silicon capping layer on $2 \mu \mathrm{m}$ thick oxide, obtained on a $500 \mu \mathrm{m}$ thick silicon substrate), following a standard procedure. An SOI chip is cleaned in acetone and isopropanol for $5 \mathrm{~min}$ in a ultrasonic bath and carefully dried with a nitrogen flow. The samples are then spin coated with $\mathrm{a} \simeq 350 \mathrm{~nm}$ thick ZEP photoresist and baked for $10 \mathrm{~min}$ at $180 C$. The PhC pattern is written on the resist, using a LEO/RAITH electron beam lithographic system, with a dose of $50 \mathrm{mC} / \mathrm{cm}^{2}$. After exposure, the samples are developed at $23 \mathrm{C}$ for $45 \mathrm{~s}$ using xylene and the pattern transferred on the silicon capping layer with a reactive ion etching step. This step was realised with a custom made system, using a $50: 50$ blend of $C H F_{3}$ and $S F_{6}$ gases. The residual resist was removed with tricholorethylene and the sample cleaved for end fire optical inspection. Typical samples are shown in fig. 1. Panels a) and b) show respectively scanning electron microscope (SEM) figures of resonators with square and stadium shape and increasing areas. These shapes were chosen because they are known to exhibit a non-chaotic and fully chaotic behaviour, respectively. ${ }^{9}$ The resonators were coupled to the external world by waveguides, obtained by removing a single row of holes in the $\mathrm{PhC}$ and coupled to high contrast channel waveguides. The position of the input and output waveguides was offset to avoid enforcing artificial symmetry constraints. The panels c) and d) of fig. 1 show a closed up view of a stadium shaped resonator and of the coupling region, respectively. In panel d) it is possible to distinguish the hexagonal periodic array of holes of the $\mathrm{PhC}$. The filling factor of the $\mathrm{PhC}$ was kept around the value $r / \Lambda=0.3$, where $\mathrm{r}$ is the radius of the holes and $\Lambda$ is the lattice constant of the $\mathrm{PhC}$, to keep the band gap in the $\lambda=1550 \mathrm{~nm}$ region. 

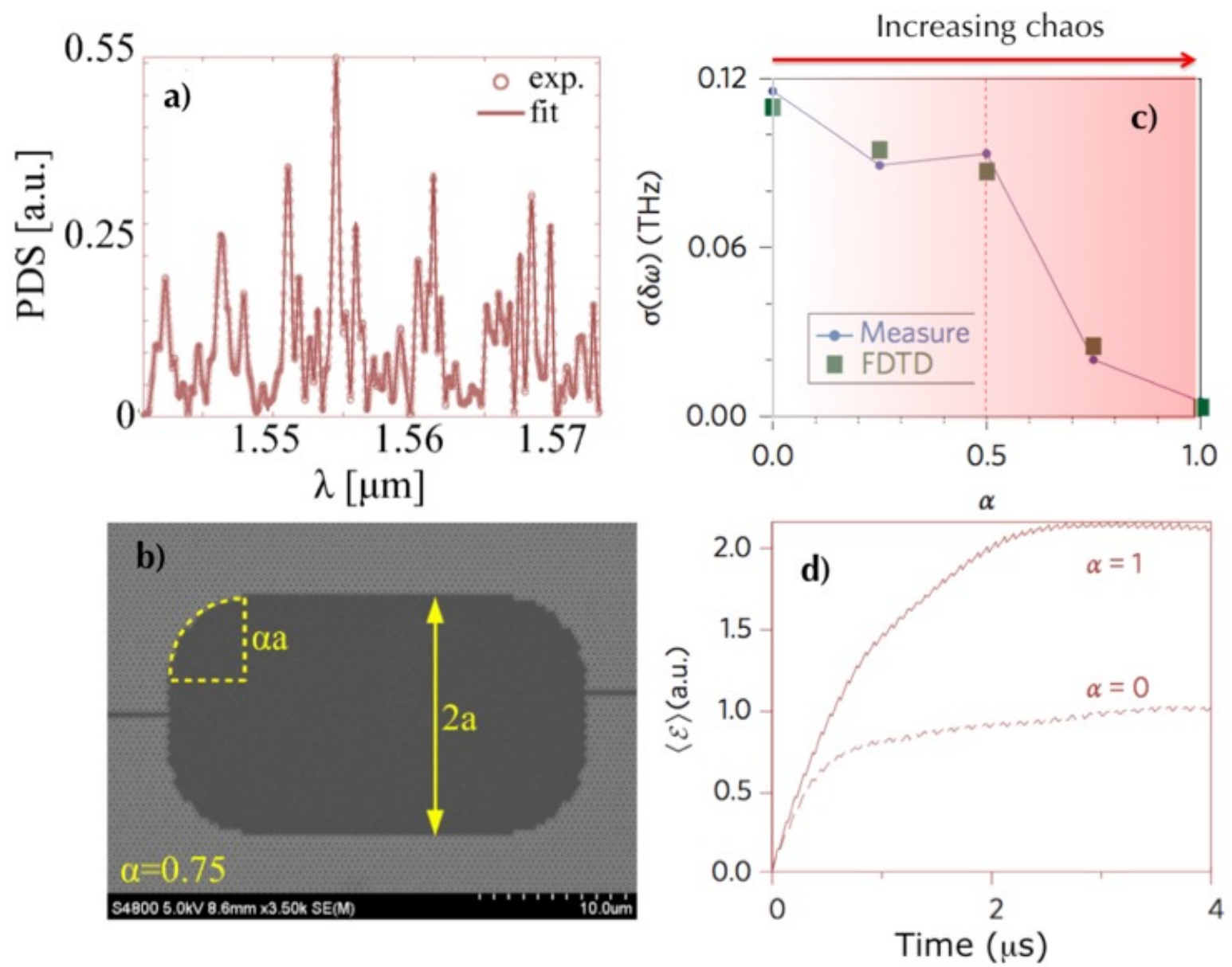

Figure 2. a) Typical power density spectrum (PDS) and reconstred fit. b) SEM figure of a PhC cavity with intermediate value of $\alpha$. c) Measured and calculated difference of max and min lifetimes of the modes supported in the PhC cavities. d) Average value of the energy stored in the resonators for different values of $\alpha$, using a light source with a bandwidth of $110 \mathrm{~nm}$.

To characterise the optical properties of the cavities we measured their transmission spectra using an amplified spontaneous emission source centred at $\lambda=1550 \mathrm{~nm}$ and of bandwidth $110 \mathrm{~nm}$ and an ANDO optical spectrum analyser with resolution below $0.1 \mathrm{~nm}$. A typical transmission spectrum is shown in panel a) of fig. 2, along with there reconstructed fit, to create a statistic of the mode lifetimes. ${ }^{9}$

\section{CHAOS AND BROADBAND LIGHT HARVESTING}

The ability to create accurate statistics for the linewidths of the resonant modes is pivotal to ascertain the chaotic behaviour of the resonators. To correlate the shape of the resonators with a specific statistic, we fabricated a number of resonators with varying geometrical parameter $\alpha$, as in fig. 2. For $\alpha=0$ the cavity is square (non-chaotic), for $\alpha=1$ it is a stadium (chaotic). Intermediate values permit to explore the full dynamics, hence $\alpha$ plays the role of a chaos parameter. The main effect of increasing the chaos is to produce the collapse of the lifetimes of all the modes supported by the resonators towards a single value (see panel c) of fig. 2 , where the difference between the max and min lifetime tends to zero). ${ }^{10}$ This, along with the fact that in this condition energy is distributed along all the available degrees of freedom is the key factor that yields to an enhanced energy harvesting efficiency of a chaotic resonator compared to a standard one. In other words, light 

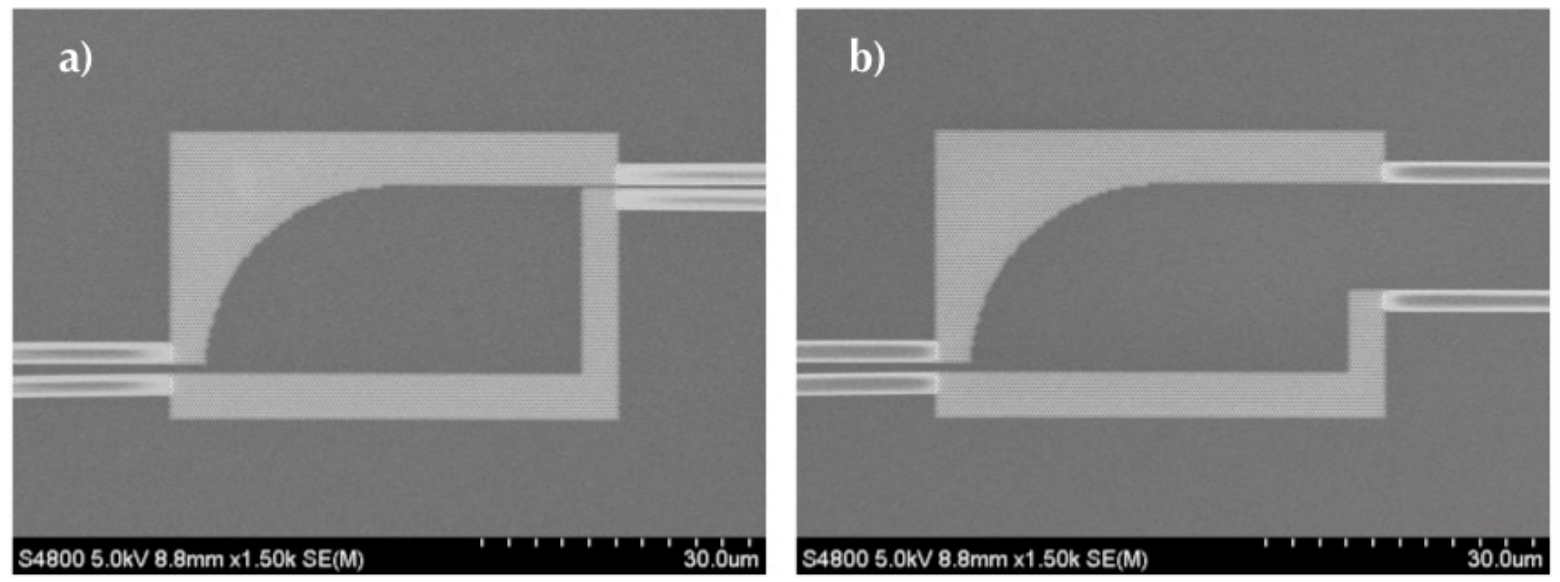

Figure 3. SEM figures of PhC chaotic resonators with output waveguide made of 1 missing row of holes (panel a) ) and 30 rows of holes (panel b) ).

can be more efficiently coupled into a system that supports many modes all with the same lifetime. This result was also confirmed by numerical simulations, comparing the two extreme cases, as shown in panel d) of fig. 2 .

\section{LOSS CONTROL AND HAMILTONIAN SIMULATOR}

The main advantage of using a $\mathrm{PhC}$ platform is that all the losses mechanism can be well accounted for. These include the out of plane losses, the losses due to scattering from the $\mathrm{PhC}$ and the input and output waveguide couplers. This permits an extremely accurate modelling and numerical reproducibility, for example using finitedifference time-domain codes and random matrix theory. This peculiar property can be further exploited by tuning the losses mechanism on purpose to explore their effect on the chaotic behaviour of the $\mathrm{PhC}$ resonator. The easiest way to achieve this goal is by modifying the with of the output waveguides. For example, panel a) of fig. 3 shows a chaotic $\mathrm{PhC}$ resonator with a single line defect $\mathrm{PhC}$ waveguide, whereas panel $\mathrm{b}$ ) shows the same system with an output waveguide obtained removing 30 holes from the regular $\mathrm{PhC}$. The main effect of these increased losses is to perturb the system and break its simple chaotic regime. Interestingly this mechanism can be used to mimic several physical system that can be described by the same perturbed Hamiltonian. ${ }^{11}$ For example, by increasing gradually the width of the output waveguide it is possible to observe the onset of supperradiance, as witnessed the by the creation of a finite number of short-lived modes. This corresponds to a system of two-level atoms coupled to a common radiation field with a strength comparable to the energy separation of their atomic level, as predicted by Dicke. ${ }^{12}$ Fig. 4 shows the map of resonance widths of the supported modes and their frequency for different defect waveguides. For high losses the superradiant modes with short lifetimes are separated from the long living modes by a frequency gap that depends nonlinearly from the width of the channels.

\section{CONCLUSIONS}

In conclusion we have shown that chaotic $\mathrm{PhC}$ resonators exhibit a very rich physics. The possibility to account for and tailor accurately the loss mechanisms is useful both for the demonstration of practical devices and the validation of fundamental theories of difficult experimental realisation. In particular we have shown that chaotic $\mathrm{PhC}$ resonators can be used to double the light harvesting efficiency over a band of $110 \mathrm{~nm}$. We have also shown that by fine-tuning the losses it is possible to demonstrate the onset of superradiant states associated to the Dicke transition. 


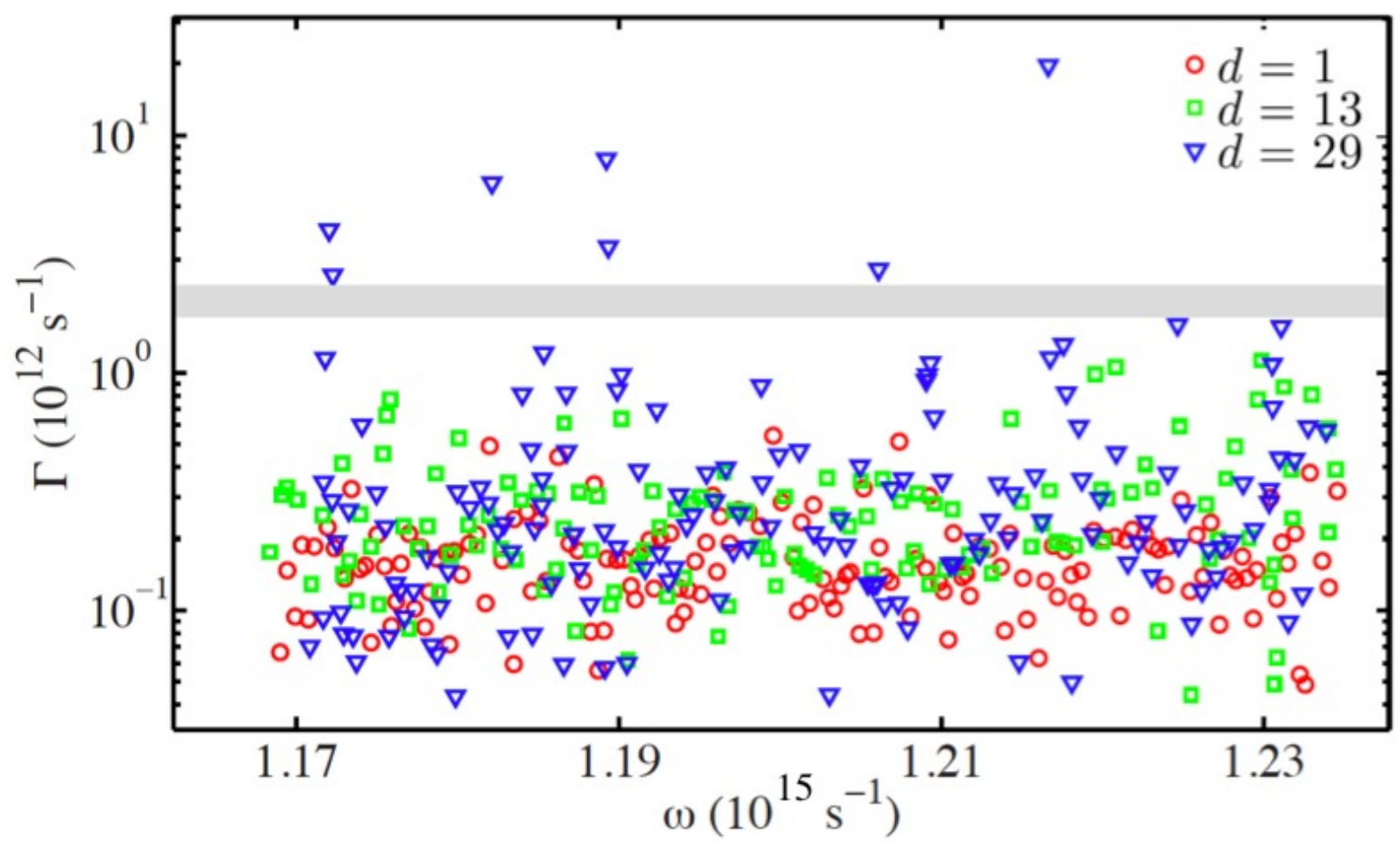

Figure 4. Map of linewidts and frequency of the modes supported by $\mathrm{PhC}$ cavities with different widths of the output channel waveguides.

\section{ACKNOWLEDGMENTS}

We acknowledge support from the EPSRC (ADF, Fellowships No. EP/I004602/1 and No. EP/J004200/1) and KAUST (AF, Grant No. CRG-1-2012-FRA-005).

\section{REFERENCES}

[1] Di Falco, A., O'Faolain, L., and Krauss, T. F., "Chemical sensing in slotted photonic crystal heterostructure cavities," Applied Physics Letters 94, 3503 (Feb. 2009).

[2] Melloni, A., Morichetti, F., Ferrari, C., and Martinelli, M., "Continuously tunable 1 byte delay in coupledresonator optical waveguides," Optics Letters 33, 2389-2391 (Oct. 2008).

[3] Shakoor, A., Lo Savio, R., Cardile, P., Portalupi, S. L., Gerace, D., Welna, K., Boninelli, S., Franzò, G., Priolo, F., Krauss, T. F., Galli, M., and O'Faolain, L., "Room temperature all-silicon photonic crystal nanocavity light emitting diode at sub-bandgap wavelengths," Laser 6 Photonics Reviews 7, 114-121 (Sept. 2012).

[4] Di Falco, A. and Assanto, G., "Tunable wavelength-selective add-drop in liquid crystals on a silicon microresonator," Optics Communications 279(1), 210-213 (2007).

[5] Di Falco, A., Conti, C., and Assanto, G., "Impedance matching in photonic crystal microcavities for secondharmonic generation," Optics Letters 31(2), 250-252 (2006).

[6] Notomi, M., Kuramochi, E., and Tanabe, T., "Large-scale arrays of ultrahigh-Q coupled nanocavities," Nature Photonics 2, 741-747 (Nov. 2008).

[7] Martins, E. R., Li, J., Liu, Y., Zhou, J., and Krauss, T. F., "Engineering gratings for light trapping in photovoltaics: The supercell concept," Physical Review B 86, 041404 (July 2012).

[8] Ganapati, V., Miller, O. D., and Yablonovitch, E., "Light Trapping Textures Designed by Electromagnetic Optimization for Sub-Wavelength Thick Solar Cells," arXiv.org (July 2013). 
[9] Di Falco, A., Krauss, T. F., and Fratalocchi, A., "Lifetime statistics of quantum chaos studied by a multiscale analysis," Applied Physics Letters 100(18), 184101 (2012).

[10] Liu, C., Di Falco, A., Molinari, D., Khan, Y., Ooi, B. S., Krauss, T. F., and Fratalocchi, A., "Enhanced energy storage in chaotic optical resonators," Nature Photonics 7, 473-478 (Apr. 2013).

[11] Liu, C., Di Falco, A., and Fratalocchi, A., "Dicke Phase Transition with Multiple Superradiant States in Quantum Chaotic Resonators," Physical Review X 4, 021048 (June 2014).

[12] Baumann, K., Guerlin, C., Brennecke, F., and Esslinger, T., "Dicke quantum phase transition with a superfluid gas in an optical cavity," Nature 464, 1301-1306 (Apr. 2010). 